\title{
BMJ Open Experiences and perceptions of patients with psoriatic arthritis participating in a trial of faecal microbiota transplantation: a nested qualitative study
}

\author{
Maja Skov Kragsnaes (D) , ${ }^{1}$ Shaun Theodor Sødergren (D) ,, Jens Kjeldsen (iD , ${ }^{3}$ \\ Hans Christian Horn, ${ }^{1}$ Heidi Lausten Munk, ${ }^{1}$ Jens Kristian Pedersen, ${ }^{4}$ \\ Camilla Schufri Klinkby, ${ }^{1}$ Maarten de Wit (D) , ${ }^{5}$ Nanna Gram Ahlmark (D) ,2 \\ Tine Tjørnhøj-Thomsen, ${ }^{2}$ Torkell Ellingsen (i) ${ }^{1}$
}

To cite: Kragsnaes MS Sødergren ST, Kjeldsen J, et al. Experiences and perceptions of patients with psoriatic arthritis participating in a trial of faecal microbiota transplantation: a nested qualitative study. BMJ Open 2021;11:e039471. doi:10.1136/ bmjopen-2020-039471

- Prepublication history and additional material for this paper is available online. To view these files, please visit the journal online (http://dx.doi.org/10. 1136/bmjopen-2020-039471).

MSK and STS are joint first authors.

Received 16 April 2020 Revised 26 January 2021 Accepted 18 February 2021

Check for updates

(c) Author(s) (or their employer(s)) 2021. Re-use permitted under CC BY-NC. No commercial re-use. See rights and permissions. Published by BMJ.

For numbered affiliations see end of article.

Correspondence to Dr Maja Skov Kragsnaes; d063631@dadlnet.dk

\section{ABSTRACT}

Objectives Patients' first-hand experiences of faecal microbiota transplantation (FMT) performed in a

rheumatological care setting have yet to be elucidated. The objectives were to explore participants' perceptions of being part of an FMT trial thereby identifying potential trial participation effects and enlightening the patient perspective on the outlook for future FMT trials in rheumatic diseases. Design In a qualitative study nested within a double-blind, randomised, placebo-controlled trial (RCT) testing FMT as a potential new antirheumatic treatment, semistructured telephone interviews were conducted following the trial participants' final 26-week visit. Qualitative researchers, who did not take part in the main trial, performed the interviews and the primary analysis. The experiences explored related to the conduct of the RCT and changes in the participants' everyday life. The analysis was carried out using a thematic approach.

Setting A Danish rheumatology university outpatient clinic with nationwide inclusion.

Participants The study included 10 patients with psoriatic arthritis (PsA) who were unaware of their treatment allocation (FMT/sham transplantation) and completed the final 26-week trial visit.

Results Participation in the RCT influenced the patients' understanding of PsA and induced positive changes in their everyday life. Renewed hopes for the future in addition to a feeling of enhanced care contributed to significant trial participation effects. FMT was deemed a tolerable and safe treatment. Conclusions Discrepancies between the clinical and the research setting should be considered when discussing the clinical relevance of the results of the RCT. Overall, patients with PSA who have participated in an RCT testing FMT find the treatment acceptable and safe encouraging more research into the field of microbiota-targeted interventions in rheumatic diseases.

Trial registration number NCT03058900; Pre-results.

\section{INTRODUCTION}

Psoriatic arthritis (PsA) is a chronic immunemediated disease of unknown origin associated with limited quality of life and increased
Strengths and limitations of this study

- This is a qualitative study nested within a doubleblind, randomised, placebo-controlled trial testing faecal microbiota transplantation (FMT) as a potential new antirheumatic treatment.

- This is the first study to investigate FMT trial experiences of patients with rheumatic diseases and explore elements of ethics, safety, concerns, hopes and impact on everyday life in relation to receiving FMT in a trial setting.

- There was sufficient repetition of ideas in data to suggest data saturation.

- A limitation of the study is that we only conducted telephone interviews one time with each participant following the final 26-week trial visit.

- The 10 trial participants' view on FMT does not necessary reflect the opinion of most patients with psoriatic arthritis.

mortality. ${ }^{1}$ Despite the new possibilities of targeted medical drugs, ${ }^{2}$ PsA remains a significant treatment challenge. ${ }^{3}$ Therefore, testing new therapies targeting other potential mediators of the disease is highly needed. ${ }^{4}$ Intriguingly, an association between PsA and dysbiosis of the intestinal bacterial community has been uncovered. ${ }^{5}{ }^{6}$ If this dysbiosis plays a role in the pathogenesis of PsA, restoring healthy diversity to the gastrointestinal microbiota could be a potential new target of antirheumatic treatment. ${ }^{7}$ One way to achieve this is by performing a faecal microbiota transplantation (FMT) ${ }^{8}$ Still, patients' perspectives of the use of FMT in rheumatic diseases have not previously been reported.

In 2017, we initiated the first double-blind, randomised, controlled trial (RCT) examining safety and effectiveness of one FMT 
performed on patients with PsA. ${ }^{9}$ The aim of the trial was to manipulate the gastrointestinal microbiota thereby seeking to reduce the overall disease activity evaluated after 26 weeks. Since microbiota-targeted therapies in general and FMT in particular suggest a new paradigm for treating inflammatory rheumatic diseases, we found it vital, from both a safety and ethical perspective, to get insight into the participants' experiences and perceptions. Hence, we chose to conduct a qualitative study nested within the RCT by performing semistructured interviews with 10 participants following their last trial visit. ${ }^{10}$ Indeed, a qualitative approach is appropriate when endeavouring to obtain in-depth knowledge about patients' perspectives and to identify potential trial participation effects. ${ }^{1112}$

\section{METHODS}

\section{Methodological guidelines and definitions}

The reporting guideline for qualitative research Consolidated Criteria for Reporting Qualitative Research ${ }^{13}$ endorsed by the Enhancing the QUAlity and Transparency Of health Research (EQUATOR) network has directed the reporting of this study (online supplemental file 1). In the following, we use the term 'RCT' when referring to the clinical trial testing FMT while the term 'study' refers to the qualitative study nested within the RCT.

\section{Design of the RCT}

The RCT commenced in May 2017 and 31 participants were enrolled until 31 December 2019. ${ }^{9}$ Patients with PsA with at least three swollen joints despite a minimum of 3 months of methotrexate (MTX) treatment, a classic first-line disease-modifying antirheumatic drug administered as weekly pills or injections, were eligible for enrolment. The trial consisted of an experimental intervention, either FMT or a sham transplantation (hereinafter referred to as the RCT intervention), being performed at baseline. We installed the microbiota transplant or the sham transplant in the upper intestine using gastroscopic guidance. Both the participants and the rheumatologists treating them remained blind to the allocation of the intervention until the end of the final 26-week visit. All patients continued treatment with MTX (maximum tolerable dose, $15-25 \mathrm{mg} /$ week) throughout the trial. The FMT transplant was donated from healthy volunteers at the local blood bank facility, who had to pass an extensive screening programme before their processed stool was released for FMT. ${ }^{14}$ The RCT design is described in more detail in the published protocol. ${ }^{9}$

Before enrolment, interested trial candidates received written and verbal information about each RCT visit, the nature of FMT, the donor selection procedure, the known short-term risks, and the limited data on longterm risks. Furthermore, all candidates had to attend a clinical screening visit where the criteria for eligibility were assessed by an experienced rheumatologist. In accordance with current treatment guidelines, ${ }^{2}$ candidates were also informed about other treatment options than FMT, including biologics. This was important information since all participants had failed to respond adequately to MTX treatment and, consequently, needed treatment adjustment to achieve the main treatment target, remission.

All participants received contact information including direct phone numbers and email addresses both to the RCT coordinator (MSK) and to the RCT nurse (CSK). Participants were encouraged to use the contact information if they had any questions/uncertainties related to their RCT participation. In case of any unexpected events, participants were immediately given an appointment to see their trial rheumatologist. As additional security, participants were told that they could on request be given an appointment with their trial rheumatologist within a few days in the event of worsening of their condition. Treatment non-responders were offered add-on treatment and/or change in their ongoing treatment if deemed necessary based on the current guidelines. ${ }^{2}$

\section{Design of the nested qualitative study \\ Research team}

The core research team comprised two senior qualitative researchers specialised in anthropology (TT-T) and sociology of religion (NGA), a qualified qualitative researcher with a university degree in public health science (STS), and two physicians (MSK and TE) who had initiated the RCT; TE being an experienced rheumatologist.

\section{Roles and relations in the research process}

None of the participants (nor the interviewer (STS)) knew about their respective RCT intervention allocation at the time of the interview. Hence, the participants' answers and reflections were unaffected by any knowledge of the nature of the RCT intervention. Nor did the participants know the interviewer prior to the initial interview contact, except in one case where the interviewer had been observer at an RCT visit. We thereby sought to create an atmosphere where participants would feel comfortable expressing their views freely about the conduct of the RCT and their personal experiences. The intention was to minimise participant bias; for example, avoiding responses that participants thought were appropriate or more acceptable answers according to the trialists.

\section{Participants}

Because of limited resources and time, we initially decided to recruit participants for the qualitative study among patients who attended their final 26-week RCT visit between February and June $2018(\mathrm{n}=11)$. Ten consecutive participants were contacted by the RCT coordinator (MSK). The eleventh participant was not contacted due to ethical grounds as he missed his final visit for personal reasons. They all consented to the interview (ie, zero non-responders) and gave permission for the interviewer (STS) to contact them by email and phone. Based on these first 10 interviews, the qualitative research team deemed that there was data saturation and that the 


\begin{tabular}{|c|c|}
\hline Characteristic & Total $(n=10)$ \\
\hline Female sex, no (\%) & $7(70 \%)$ \\
\hline Age, year & $53.9(11.3)$ \\
\hline Time since diagnosis, year.* & $7.4(4.2,12.8)$ \\
\hline Rheumatoid factor IgM negative, no (\%) & $10(100 \%)$ \\
\hline $\begin{array}{l}\text { Anti-citrullinated peptide antibody } \\
\text { negative, no (\%) }\end{array}$ & $10(100 \%)$ \\
\hline HLA-B27 negative, no (\%) & $8(80 \%)$ \\
\hline C-reactive protein, $\mathrm{mg} / \mathrm{L}$ & $4.5(3.6)$ \\
\hline HAQ-DI† & $1.09(0.40)$ \\
\hline Swollen joint 66 count & $7.4(3.8)$ \\
\hline Tender joint 68 count & $22.8(7.9)$ \\
\hline \multicolumn{2}{|l|}{ SPARCC enthesitis index $\ddagger$} \\
\hline Score $\geq 1$, no (\%) & $10(100 \%)$ \\
\hline Score in patients with a score $\geq 1$ & $8.7(4.0)$ \\
\hline
\end{tabular}

Data are mean (SD) or $\mathrm{n}(\%)$ unless otherwise stated.

*Time since diagnosis of psoriatic arthritis is presented as median and IQR.

†Scores on the Health Assessment Questionnaire Disability Index (HAQ-DI) range from 0 to 3 , with higher scores indicating greater disability.

¥Spondyloarthritis Research Consortium of Canada (SPAARC) Enthesitis Index range from 0 to 16 , with higher scores indicating more severe disease.

sample of 10 participants was sufficient for the qualitative analysis and the scale of this study. Characteristics of the 10 participants are presented in table 1 .

\section{Interview method}

Semistructured telephone interviews were conducted using an interview guide (online supplemental file 2) focusing on the participants' thoughts and considerations at four different periods of time: (1) before RCT participation; (2) after the FMT/sham intervention; (3) during RCT participation; and (4) right before the end of RCT participation. Issues covered in the interview guide were thoughts and motivations behind joining the RCT, the view of FMT as a potential new treatment of PsA, reflections about side effects, risks and benefits of FMT, concerns about receiving a sham procedure, experiences in relation to the conduct of the RCT, changes in everyday life, and the outlook for future trials testing FMT. The semistructured interview allowed the interviewer to follow-up with probes and more open-ended questions when participants expressed issues of importance to them that were not addressed in the interview guide.

The interviews were conducted between April and September 2018. Median time from the last RCT visit to the qualitative interview was 66.5 days (range: 4-216). All interviews began with an introduction, during which the interviewer familiarised the participants with the overarching themes of the interview guide, the objective of the research, and how the results would be used and published. Interviews were audio recorded with participant consent and subsequently transcribed verbatim and anonymised. Transcripts were checked for accuracy by STS. The interviews lasted on average $35 \mathrm{~min}$ (range: 20-42 min).

\section{Analysis}

The analysis was a team effort of the interviewer, the qualitative researchers, and the two physicians participating in the RCT (investigator triangulation). The interviewer (STS) and the two independent qualitative researchers (TT-T, NGA) conducted the primary analysis using a thematic approach. ${ }^{15}$ Initially, through a process of open coding, they identified the preliminary themes from the 10 interviews. Themes and subthemes were refined through collaborative analysis meetings (TT-T, NGA, STS, MSK, TE) and subsequent focused coding of the material. $^{16}$

\section{Patient and public involvement}

In the planning stage of the study, both patients who participated in the RCT and those who were not eligible or decided not to participate gave input to relevant issues and specific questions that were addressed in the interview guide. Areas of priority stressed by the patients were impact on everyday life and risks and benefits associated with RCT participation. A patient partner (MdW) who did not participate in the interview nor in the RCT commented on the analysis and the manuscript. He made suggestions for improving the reporting of the study method and assisted the interpretation of results.

\section{RESULTS}

\section{Central themes}

In the following section, we describe four main themes, which we found central in participants' accounts of their participation in the RCT. These are:

1. Motivation for participation. This refers to elements of decision-making involved in taking part in the RCT.

2. Impact of trial-related activities. This refers to elements of the RCT that were not codified in the protocol but turned out to have significant implications for participants' experiences of the treatment being tested.

3. Changes in everyday life. This concerns the changes that participants experienced physically, psychologically, and socially during the RCT.

4. Receiving FMT. This addresses participants' concerns about safety, discomfort, and side effects relating to FMT, before, during and after receiving the treatment.

\section{Motivation for participation}

This first theme focuses on the participants' reasons for enrolment in the RCT. In the patient stories, we distinguished two motivational factors, namely renewed hopes of new and better treatments, and the inclination to help others with the disease. 
Hopes of new and better treatments

The first motivational factor was the hope of getting better as a result of new treatment options for PsA. These hopes were related to the severity of participants' condition, information from the media and the natural element of FMT. For one thing, all participants were eager to get better because they were severely affected by their condition when they entered the RCT and had failed to respond adequately to their current MTX treatment. During the interview, many participants recounted that they were influenced by pain to such an extent that they could not carry out a normal job, participate in sports, or take part in family gatherings. As one participant explained: "I had a period where I was practically like a bit of a zombie, you go and carry out your work, you look after your family and all like a routine, you do what you have to, and then you don't have much energy for all sorts of other things. I used to play elite-level handball and was a trainer and had what it took to do a whole lot of other things, and suddenly I just didn't have anything left when I started to suffer from my arthritis and all that". The hopes of improvement was expressed in statements such as: "I was hoping that I would get better, I mean that I would have less pain and more mobility... that I would get relief somehow or other", and "it's obvious that, when you're part of some research, you'd want to get the thing [experimental trial intervention] that is supposed to ensure you get better".

For some of the participants, the positive expectation arose from what they had heard about FMT in the media. As one participant explained: "I had heard it on the TV news. It was with people who had chronic diarrhoea and it worked on them." Other participants became aware of FMT through television talkshows "I had seen something on television, on some breakfast tv about a donor and a patient who had met up. And it was really, really informative", or through documentaries: "I saw a programme on the television about some modern miracles... and there was certainly one youngish fellow who was really in a bad way and who got much better from having those gut bacteria replaced". As seen from the quotes, trust in or curiosity about the treatment came from various news channels. Many participants could relate to stories of patients suffering from other chronic diseases. Their positive experiences with FMT gave the participants hope. As one elaborated: "They got better, you know, and just think, if they got better, then maybe people like us might be able to as well".

The hope of getting better was also related to the idea of FMT being a natural, and therefore a favourable alternative to established treatment options: "And when I then read a bit more about it and heard a bit more about it, I thought that as a whole it sounded interesting in the sense that you didn't fill yourself with all sorts of medicine but with something that was natural already". Several participants also used the term 'hocus-pocus' about FMT, being uncertain about the ways the intervention might affect them, since it was not a conventional synthetic medical drug but, in contrast, a biological material. The hocus-pocus phrase was used either to describe FMT as an alternative treatment or to distinguish FMT from being a hoax: "It wasn't as if they started out on some complete hocus-pocus or other, because it was a method of treatment that had been used in other contexts". Hence, even though FMT was not viewed as a medical drug, the intervention was considered a reliable treatment alternative.

The participants' perception of FMT being a natural treatment-and therefore better than conventional medical drugs - was reinforced by their disappointment with the effects of their current MTX treatment: "For a long time I took it [MTX] and it had no effect", In addition to their disappointment with MTX due to its lack of effect, several participants described it as an unnatural and toxic drug: "I reacted badly to a lot of the medication I have taken, so you might say that means that fortunately a ... how should I put it, a natural (giggles) treatment would be preferable", "I would much rather have a natural product than I would take all that poison, because there are loads of side effects from the other [MTX]", and "if you can work with the body in a natural way, without having to take this strong medication [MTX], then I can't see that I have anything to lose in doing so".

Despite thorough written and verbal information about the nature of FMT and the fact that the treatment had not previously been tested on patients with rheumatic diseases, none of the participants expressed concerns that the FMT might worsen their arthritis. Instead, hopes for improvement overshadowed any potential negative effects: "I had nothing to lose in doing it, I thought, since I couldn't get any worse anyway", "not worries, only hopes". In addition, the participants felt that they were well informed and trusted that the health professionals at the hospital would not set up a trial that they did not perceive to be safe: "I may be a little naive, and I may just be a very positive person, but I think that if they are doing this it is probably because they have a sense that it might work and the side effects that there might be would have been weighed against the effect they think they might gain from it. So, really that isn't something that concerns me". Overall, these statements show that a dominant theme among participants was the hope for new treatment and little consideration of unknown risks.

\section{Helping other patients}

Another motivational factor behind their participation in the RCT was the inclination to help other patients with PsA by contributing to research and knowledge in the field. For many, the RCT provided an opportunity to help improve treatment of the disease or, as one participant put it: "right at the beginning, like, it sounds just so disgusting, but when you get to read it properly, then I think actually it is exciting to say that we have contributed to some research... that we have done something for the disease we happened to have that might help them on the way to find some other possible ways for curing itthat is part of what counts most heavily for me". Some 
participants felt that having the disease laid a special responsibility on them. This was evident from expressions such as "obligation to participate in something like this", "importance of supporting research", and "contributing whatever we can".

The prospect of helping other patients in the same situation as themselves found expression in statements as "it's a good thing, too, to take part in a trial like this, which might even be able to help other people", "it sounds incredibly interesting, that you might be able to help a whole lot of arthritis patients through such a small intervention", "in truth, I hope that other can benefit from it, too". All participants were told that they had a $50 \%$ risk of undergoing a sham procedure and not getting any donor bacteria. Nevertheless, they were willing to participate, arguing that the RCT results could help others in the future: "Well, if it doesn't help me then maybe it might help the next person who gets PsA", and "if getting those gut bacteria can contribute to helping people who have a disease that is as goddam nasty as this one, then I'm happy to have been a placebo as regards whether it works or doesn't work". These quotes illustrate that the participants felt that their disease brought with it an obligation to contribute to knowledge in the field, if possible. Most participants also expressed a sense of responsibility and an empathy for others in the same situation, which only increased their motivation to act against the disease.

In conclusion, the major motivational factors for joining the RCT were the hope for recovery and the possibility of helping others suffering from PsA out of the severe state of pain and the social and relational implications of the disease. The dissatisfaction felt by most participants with their current treatment with MTX due to its lack of effect and/or to its negative side effects meant that they were highly motivated to participate in research that tested what they saw as a natural and less harmful form of treatment, which had proved effective in the treatment of other serious conditions.

\section{Impact of trial-related activities}

This second theme refers to the finding that the RCT virtually implies two interventions. The first is the planned experimental intervention of the RCT (FMT/sham). The other is the somewhat uncontrolled intervention which is made up of all the activities that are not recorded or directly accounted for as part of the RCT but, which we found, had significant implications for participants' experiences of the treatment being tested. This section will focus on two domains emerging from these activities, which, while they have little bearing on the primary purpose of the RCT - the results of FMT — are highly significant for the experience of participants, namely recognition and care, and maintaining hopes.

\section{Recognition and care}

A key feature deriving from the trial-related activities was the sense of recognition and attention felt by participants. This encompassed a number of positive implications of
RCT participation such as "feeling safe", "being taken care of", "being listened to", and "being taken seriously". Indeed, most participants judged to some degree that they had experienced an improvement in treatment and care compared with their usual encounters with the healthcare system. This experience was clearly expressed in the following statements: "everything is examined, everything in my body and everywhere ... from one end to the other", and "I have felt very positive about my contact with the rheumatologists in Odense, and I don't think I have ever been examined as thoroughly as I was in Odense [in relation to participation in the trial]".

Furthermore, the participants found that the health professionals took the time to examine and listen to them during the RCT visits: "I mean, personal relations really mean so much, the way you are received, there being time for you...", and "that there are some things they going into more deeply compared to what normally happens, because it can seem superficial when it's just a matter of a 5-minute check like that and then out the door again, you know, but here there's more, as a patient they go into more depth when it's a trial". Another participant experienced an immediate improvement in her condition, which only lasted 3 days. She attributed the brief improvement in her condition to the heightened attention by the health professionals at the beginning of the RCT: "that suddenly attention is being paid to my disease just around the time when I was given the transfer, that has had psychological significance, too". Other participants experienced no change in care: "I don't think they've been keeping more of an eye on me than they have done otherwise ... I always feel I am treated well".

During the RCT, participants also felt that the health professionals acknowledged the severity of the disease, and that their symptoms, limitations and challenges of everyday life were taken seriously. By contrast, before their RCT participation, they had often felt misunderstood and had a sense of being hypochondriac. An example of this change in disease recognition is seen in the following: "I have become clearer about my disease with this project... well, it's true enough that I am ill, I have these symptoms here, it's not just something I'm saying or anything. You know, that feeling that you're just a hypochondriac? Because you can't live up to the everyday life you are used to having". These feelings were related to difficulties in accepting that they had PsA.

Being part of the RCT also increased participants' understanding of the implications of the disease in relation to their everyday life. As one participant expressed: "well, the project has also maybe got me to think differently in that I have come to accept the pain that there is". Another participant described how she had started to accept being a patient during the RCT "that it was like you achieved some clarity about some things", and that this understanding of the disease gave her a sense of relief. Participants also expressed their gratitude that the RCT included patients with PsA. This gave them a feeling that PsA was considered an important disease by 
the health professionals: "now I felt that there were some people who were at last going to take care of my arthritis and, yes, were interested in my arthritis and in me".

Alongside receiving extra time, attention and recognition from health professionals, several qualities of the contact were highlighted as important elements for the patient-physician relation including being 'good', 'professional', 'helpful', 'trustworthy', and 'informative'. These elements were seen in the following excerpts: "of course it means a lot that you have good contact", and "the people I came across were professionals, and the nurses were incredibly helpful in every way. And the doctors I had close contact with ... were incredibly informative, helpful... that gives the project quality, it means that you can believe in the project and in the people that are around it. So that gives it huge significance".

In addition, participants had a direct phone number to the RCT coordinator. Although the participants rarely used this option, they stressed it as a key feature for ensuring comfort and eliminating stress and anxiety throughout the 26-week trial: "It gives you a sense of security... you know, I haven't felt the slightest insecurity at any time". Another participant underlined the importance of continuity spending time with the same health professional and being able to share both personal and illness-related issues: "no doubt that it has had a positive effect on my participation, for I have been looked after from start to finish...". Overall, these statements stress the importance from a patient perspective of recognition, continuity, time, and quality in the relation with the health professionals, which in this case contributed to changes in the participants' disease perception and a feeling of extra care.

\section{Maintaining hopes}

A major motivational factor for joining the RCT, as presented above, was the hope for new and better treatment options. This belief in the disease-modifying abilities of FMT was maintained during the RCT. As one participant expressed it: "for all the time I was part of the trial, I had a hope that I would see an improvement in my disease". This formed a sharp contrast to their experience before their participation, where many had lost faith in the chances of a cure or amelioration of their PsA. In the words of various participants: "I have a brighter hope in my future. Yes, it will be brighter", "a miracle cure", "I hope to be able to leave treatment completely, if/when this transfer works", "curative possibilities", and "just for me to know that you don't just have to have methotrexate or biological therapy. You can also take other routes and find something that can help". Even though the health professionals did not use terms like "cure" or "alternative treatment", the mere participation in the RCT allowed the participants to envisage the potential prospect of finding a cure for the disease.

One of the participants who did not experience any major improvement in his condition maintained a strong belief in the effects of FMT: "In my way of thinking and my world, I believe that that stuff [FMT] is something that works. It may not be something that works in my particular case, but I just believe in it anyway...". Likewise, another participant reflected on the impact of the activities that accompany participation in an RCT. Although he hoped that the FMT had caused the improvement in his condition, he acknowledged that trial participation had brought about positive changes that had reinforced a positive perception of his condition, which in turn made him believe he had a better future living with the disease. "Well, I do feel better, whether that is psychological or whether it's because I have been given those gut bacteria. Right now, it doesn't matter much to me whether it's the one or the other. I just know that I feel better".

Similarly, other participants who experienced a reduction in symptoms during the RCT felt that the improvement was amplified by renewed optimism: "When you get better, you do not spend the same amount of time feeling pain and you become much more positive, right? When things get easier in your everyday life and so on, then of course your attitude changes, you want to change, so you change", "I grew even more positive about it when it began to change for the better, so I was just so pleased that I agreed to take part", "I have developed a form of optimism about my disease", "Yes, it is, like, you could say that it has opened up a hope, because it is psoriatic arthritis and that's not that easy to work with...", and "I sense that there has been an effect, and that also means that I am positive". In addition, the enthusiasm of the health professionals boosted the positive effects: "then I encountered people who also became enthusiastic for my sake and for the sake of the project, then it became, then it had, you know, such a synergy effect, which just grew even more positive". For the majority, positive attitudes towards FMT and hopes associated with possibilities of a new treatment paradigm did not abate during the trial, regardless of the effects experienced following the RCT intervention.

Interestingly, although participants who experienced positive effects acknowledged that their RCT participation could have enhanced their capabilities for coping with the disease during the trial, they did not think that the RCT participation in itself could be responsible for such major changes in their condition: "Because it was such a profound change, so I do not believe that my brain could have caused it", "because if I have not undergone it [FMT] then I don't know what has happened, because something has happened...", "if what I have been given is water, then they should really start researching into water", and "there have been many times when I have thought that if I have been given a placebo, then I can do more with my brain (laughs) than I thought I could...". These reflections show that the participants had been wondering whether the symptom relief was related to the FMT intervention or if it could be due to changes in their 'mental outlook'. Although they found the first explanation most likely, they remained open for other factors encompassed in the 'placebo effect' that 


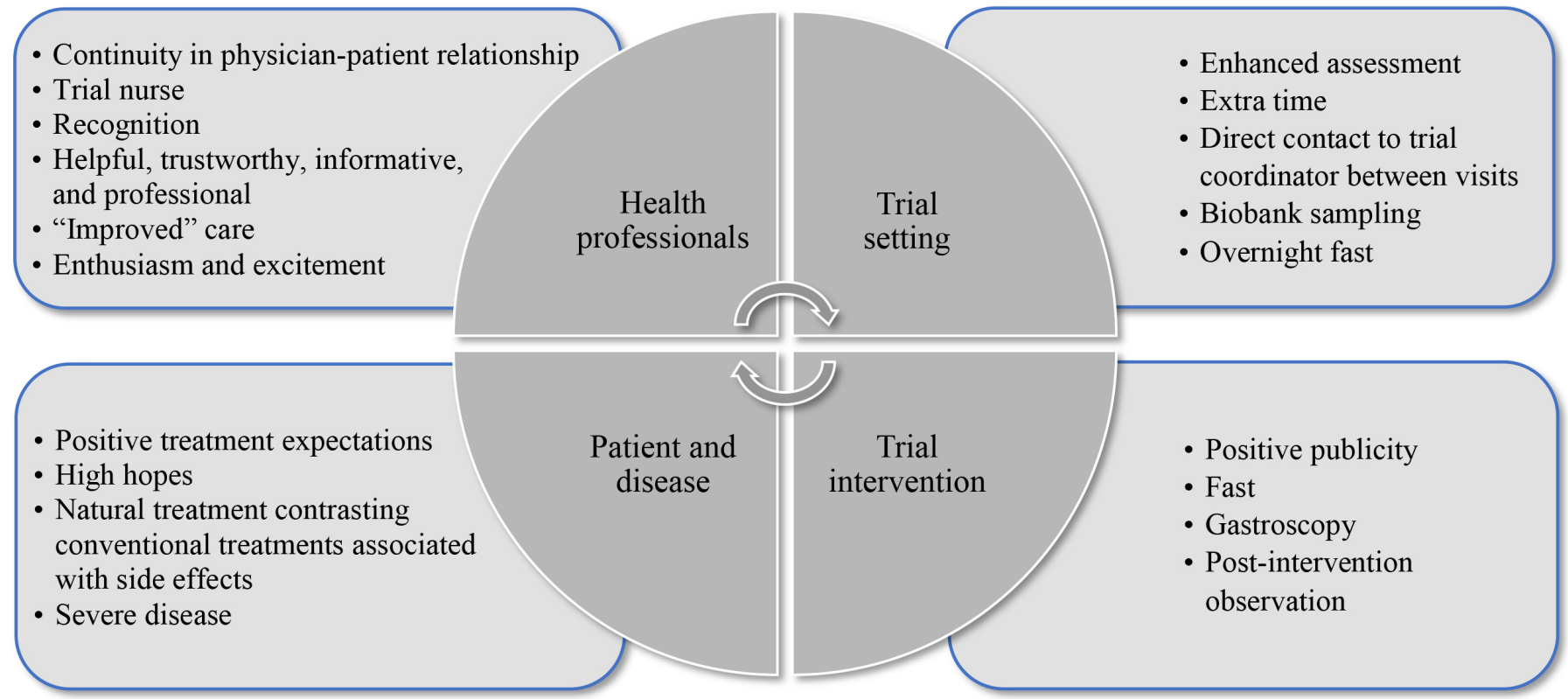

Figure 1 Factors related to the randomised controlled trial $(\mathrm{RCT})$ that may have promoted trial participation effects.

could have contributed to the improvement of their conditions.

In conclusion, the impact of the trial-related activities (see figure 1) involved two main dimensions within the affective domain: 'recognition and care' and 'maintained hope'. First, the sense that their disease was recognised altered the participants' self-image and disease perception. Second, the hope of finding better treatment options that the participant had at the beginning of the RCT did not abate during the trial, and for some, the hope was even reinforced by a reduction in symptoms, enthusiastic health professionals, and enhanced disease coping. Hence, the overall conduct of the RCT resulted in unexpected effects, which had positive implications for participants' experience of the treatment being tested.

\section{Changes in everyday life}

This third theme focuses on the changes that participants experienced in their everyday lives during their RCT participation, resulting in two subthemes; changes in physical condition, and changes in psychological condition.

\section{Changes in physical condition}

The participants experienced predominantly positive changes or no change at all in their physical condition. The changes involved reduced psoriasis and a decrease in pain as well as increased mobility and overall improvement in their function of everyday life: "My joints have become much better, I mean I have no pain in the ligaments that usually hurt, and the swelling has started to subside, and I have begun to recover much more energy again and things like that, so my arthritis has got better ... And yes, my psoriasis has also started to disappear", “... I have become more able to manage by myself than I was before".
The onset and duration of the improvement varied as illustrated in the following excerpts. Some participants experienced a rapid onset of physical improvements within hours or days of the intervention: "It didn't take more than a few hours before things started happening in my body ... got it put down on Monday and the two following days, it is indescribable, it was so amazing what happened to my body... for about the first fortnight, very positive effect", "the first two days I was sure it had worked. Then I thought it was just all brilliant, but it all faded quite quickly ... then the arthritis returned. Then I felt the pain in my joints again", "the first month I felt significantly better in a very short time ... there I went from not being well at all to being almost normal".

Even though some participants experienced a rapid change in their condition within a few days following the FMT procedure, most of the participants did not feel any noticeable differences in their condition until weeks or months after the intervention: "...after ten days I started to feel a reduction of the pain...", "Several weeks passed... my rash wasn't as irritating, it didn't itch as much...", "about a month and a half passed ... then I myself thought that I could speed things up a bit here and there", "well, I do feel much better ... it has come over time ... after two months or something like that", and "I think that some time passed before I kind of felt that I wasn't quite as tired, and I started to wonder when the next one would come, like where the pain was ... but it lasted maybe sixmonths". For others, the symptoms fluctuated, "I mean, after the transfer of the gut bacteria there were also, like, some ups and downs but nothing that lasted."

Following reduction in pain and improved mobility, some participants found they were able to do tasks that they previously had been able to do only to a limited 
extent, such as engaging socially with family and friends, doing garden work and sports: "I feel generally better, I am better able to take part in some things", "just all of a sudden I could carry my son around again and that was not something I had been able to do, not for a long time actually ... and, I mean, sometimes I would almost have to plan to play with my son just so as to have the energy to do it. I don't need to do that anymore, now we can go out and play football if we want", “... that's to say, where I hadn't been able to open jars and tins before and had to have people to help me with all sorts of things and get things down from shelves and stuff like that, now suddenly I can do it myself... at the end of December I went out and played tennis again, and I haven't been able to do that for several years", and "my fingers are smaller, I mean I have fewer swollen joints and, I can, for example, work in the garden without feeling like death for the next week because my arms can't cope with it and my fingers can't cope with it ... I usually take painkillers after I have been in the garden for just half an hour, I have had none of that this year".

In contrast to these positive experiences, two participants encountered no change in their symptoms during the RCT, and a third described how his skin psoriasis improved while his joint and tendon symptoms worsened: "I have had quite a bit of trouble with my fingers ... and my wrists. It's difficult to say because it goes up and down. Whether it is exactly because of this. There was a major effect on the pain afterwards, I mean as though... they had gone in and done something there ... that is to say, a reaction". Two of the three participants who did not experience any positive changes in everyday life function received additional antirheumatic treatment during the trial.

\section{Changes in psychological condition}

Many of the psychological changes experienced by the participants were closely related to reduction in pain and improved ability to get around without discomfort. Generally, the changes in psychological condition, whether directly related to changes in physical condition or not, included the sense of 'surplus energy' and higher activity levels, as illustrated in the quotes below: "Well, some kind of explosion, you can't describe it, for it was like there was some kind of explosion that happened in your body. And one of those nights, when I didn't sleep much, it was also as though you'd just been completely speeded, like you'd been hyped (giggles), you felt charged and you got all this extra energy, it was completely, I can't begin to describe what it felt like in your body. I mean, it was like it was working full power with positive energies and vibes, it was really crazy", "... I have got all my surplus energy back that I haven't had for ages... it was like getting my life back again ...", "all that pain stuff has all gone, it releases a mass of energy ... It has become easier because I don't have to think about my disease all the time and what I can do with my arthritis".
Like improvements in physical condition, their regained energy had a direct impact on everyday life “... That surplus energy and engagement you got, that was amazing, in fact my December was simply fantastic, just super, you know having extra energy to do things when you got back from work, to make dinner straight away, prepared cold raised buns and had extra energy and got Christmas done and a whole load of things that you otherwise couldn't...", "I got much more energy suddenly again, and I have started to do things that I haven't been able to do for ages and stuff like that", "I don't get tired quite as easily and have slowly stopped taking the morphine pills I was given and it's a really positive thing in my daily life". Family and friends also noticed positive changes: “... it's certainly something others could sense in me a lot and they say so, too, that they have been able to feel that my good humour and my energy have returned." As seen in these quotations, the participants and their relatives found that the restored spirits and energy excess had a positive impact on everyday life.

Even if the changes entailed improvement, they were not felt to be exclusively positive by all participants. One explained that she was not mentally prepared for the rapid improvement in her condition. In particularly, she felt that it was difficult to get used to her new role in the family and the ability to engage more fully in daily activities and help with the household: "I felt so well that I couldn't get used to it. My daily life was not designed to allow me to be able to do as much as I actually could. My brain had been left behind... It sounds a bit surreal, but when you have such chronic pain, everyone goes and hopes for a miracle. But no one thinks it's going to happen. So, when it does happen, you're simply not prepared for it ... I mean, the rug is just pulled away from under you. I wasn't ready for that at all and to be honest I wasn't really very pleased". This account indicates that, for some of the participants, the disease and the subsequent limitations to everyday life had become a part of their social identity and that getting better and regaining health can be difficult to adapt to. ${ }^{17}$ It may involve a longer process, which encompasses not only the physical dimension but also a mental dimension, an adaptation to being positioned and positioning oneself differently.

Finally, those participants who did not experience lasting improvements or felt that their disease was exacerbated expressed disappointment: "of course it does make you feel pretty low", "it feels, you know, a bit of a downer, if you understand what I mean, and, like, arrgh I thought that was a damned shame, but well, sod it, and then on we go". Despite disappointment with the lack of effect, they did not express great despair. Nor did they perceive increased limitations in everyday life. This could be due to their preserving hopes for the positive effects of FMT, the implication being that they had only received the sham transplantation, or that other patients in the RCT could have benefitted from the intervention-as one participant put it "then I was very interested in how it had gone with the other guinea pigs". 
In conclusion, the changes in the participants' psychological condition were interrelated with the changes they experienced in their physical condition. The combined positive changes in the physical and psychological conditions led to them having more energy and increased mobility. The onset, intensity and duration of these changes differed, but most participants experienced shorter or longer periods where they could engage more actively in everyday life and participate in activities, which they had not been able to take part in before the RCT.

\section{Receiving FMT}

The fourth theme focuses on participants' concerns about safety, discomfort and side effects relating to the FMT before, during and after undergoing the treatment. Before RCT enrolment, some participants displayed concerns about having to undergo a gastroscopy in connection with the RCT intervention. However, when asked afterwards no one felt that it was an unacceptable procedure: "The only thing I was a little nervous about was actually the transfer itself ... but that was nothing to worry about", "I didn't like the idea of having that examination [gastroscopy] but it went fine, didn't it? It was nothing really, I didn't actually feel it at all", and "being given those gut bacteria, that was a piece of cake, as it were, I mean, there was nothing to it, having them just injected...". In this context, it is worth stressing that all participants received a drug that relaxed them and induced a light sedation just before the intervention which made them become more indifferent during the gastroscopy.
Only a few had concerns about safety issues in relation to receiving stool from an unknown donor, and asked about "how much this excrement has been screened", "how do they do that? Does it have to be eaten or how will you do it? ... and whether there was something that could transmit infection and things like that?", and "what sort of gut bacteria were they? Now I had also read that the people we got bacteria from had been thoroughly screened, so I didn't have so many concerns about that as far as that went, but it is, well, it does challenge your boundaries a bit having gut bacteria fed into your own gut...". This underlines the importance of providing thorough information regarding the recruitment process of stool donors and the content of the screening programme. Only two participants reported minor side effects following the intervention. One reported, "I woke on the bed, I was fully anaesthetised, (laughing), and there I had shat myself. That was really the only discomfort I had, and I was tired, too". The other participant recounted that, "I found two days after the transfer when I ... had heartburn and diarrhoea for about an hour and that was that really. And I have had no other problems with it". The remaining participants did not report any negative side effects after the intervention.

At the end of the RCT, the participants supported the initiation of new trials to test the safety and effects of FMT and concluded that they were willing to participate in a similar trial again (see figure 2). One participant also expressed hopes for the treatment to be developed into a pill and become an approved treatment for PsA: "Then

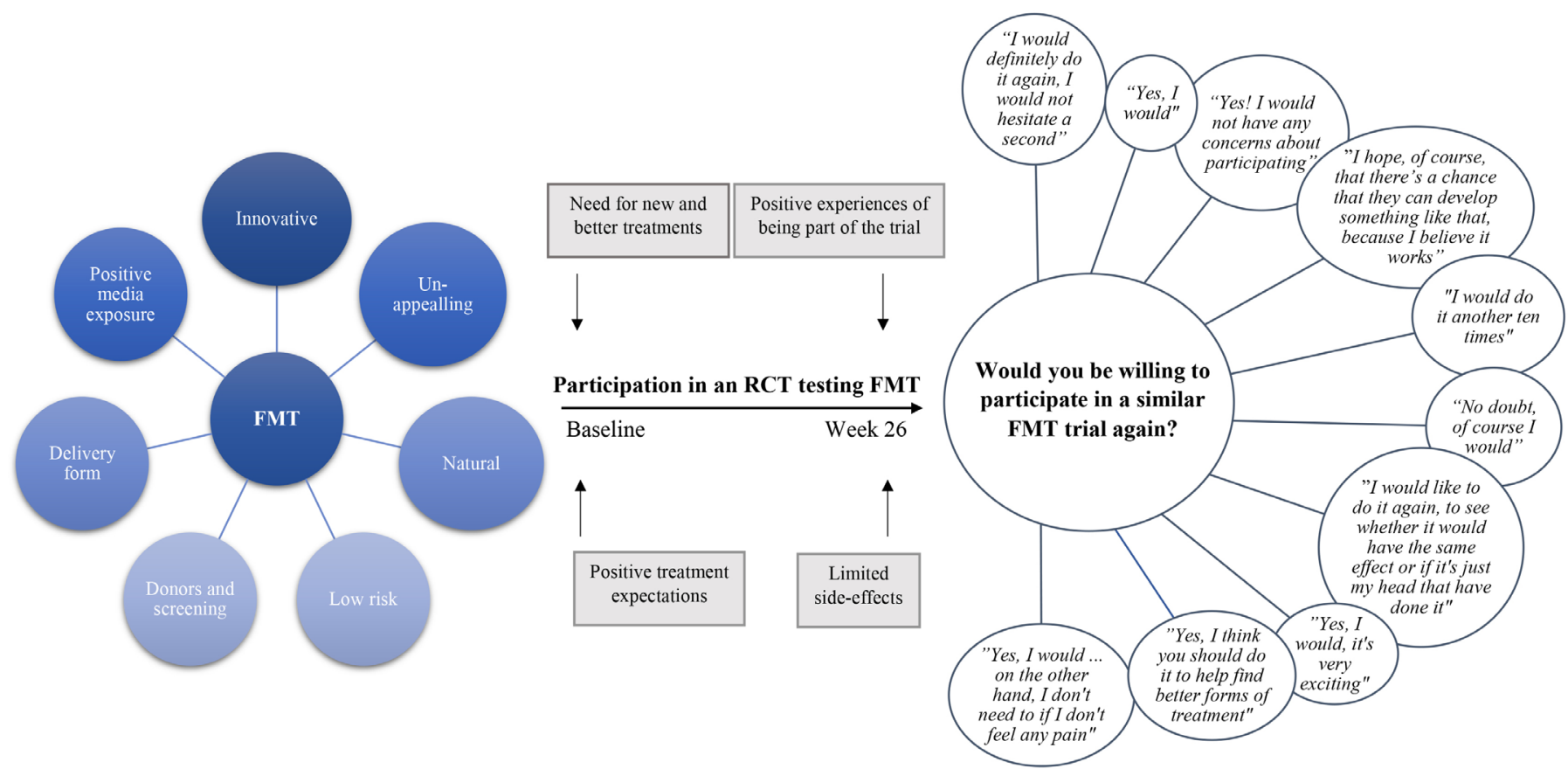

Before the RCT

After the RCT

Figure 2 Participants' view on and considerations about faecal microbiota transplantation (FMT) before and after the randomised controlled trial $(\mathrm{RCT})$. The views and considerations 'before' and 'after' the RCT were collected retrospectively at one time point at the end of the RCT, but before the participants were informed about the results. 
it would be tip-top for me if I could get away with taking these gut bacteria once in a while and if they then got so good at making them that they can be taken as a tablet". These quotes indicate that the participants felt that research into new antirheumatic treatment approachesin this case FMT-were highly important in improving the lives of patients with PsA. In conclusion, the participants reported that gastroscopic guided FMT is a tolerable procedure. Still, some expressed hopes for other types of FMT administration such as conventional pills.

\section{DISCUSSION}

As part of the first double-blind RCT of FMT conducted in patients with PsA, this qualitative study was designed to shed light on the participants' perspectives of the RCT in general, including specific experiences of trial participation and perceived responses to the intervention. The first important finding is the indication of significant trial participation effects. ${ }^{18}$ The second finding is the high acceptability of FMT and the participants' persistent beliefs in the potential of microbiota-targeted therapies.

\section{Trial participation effects}

First, participants expressed great enthusiasm for the relation with the health professionals conducting the RCT and made it clear that the general disease management during the trial had a favourable impact on their trial experience. This impact was the result of trial-related care such as extra time, frequent contact, continuity and trust. These elements were not specifically codified in the protocol indicating 'care effects' of the RCT. The mechanisms behind the effects observed in this study are not clear. However, research participation effects are well-known phenomena and may be attributed to several unconscious thoughts and actions of the participants and trialists. ${ }^{19}$ For one thing, the health professionals/ trialists (seen from the participants' perspective) may 'improve' their care of the patients/participants because of their commitments to the research project, in which it is crucial that participants do not drop out. The transferring of trialists' enthusiasm and excitement of the project to the participants is another possible contributing factor.

The observed effects of care overlap with elements of placebo effects which are inherent in most aspects of healthcare..$^{20}$ Placebo effects induce valid psychobiological effects encompassing complex mind-brain-body interactions across many conditions. ${ }^{21}$ These effects are generated and maintained by the psychosocial context around the patient involving the healthcare encounter, the doctor-patient interaction, the wording and behaviour of the health professionals, and treatment expectancies. Positive expectations on the part of the trialists also play a role in the placebo effect. ${ }^{21}$ In our study, positive treatment expectancies were identified as an important part of the theme, impact of trial-related activities, as illustrated by the persistent hopes of FMT's beneficial effects. In addition, motives for trial participation such as hope of personal disease improvement may have augmented this effect. In contrast, although nocebo effects have been reported in other rheumatological settings,${ }^{22}$ this seems not to have been a prevailing factor in this RCT.

The Hawthorne effect encompasses several elements of trial participation effects including the impact on participants' (and trialists') behaviour as a result of awareness of being assessed. ${ }^{23}$ This effect could likely have been present in the RCT as well. Indeed, many participants inquired into what they could do themselves to maintain a healthy diverse microbiota following the RCT intervention. Although they were told not to make any dramatic changes in their lifestyle in relation to diet and exerciseas we wanted only to introduce one major intervention in the RCT (ie, FMT) - their eagerness to do something extra to achieve a beneficial trial outcome may have contributed to the observed changes in disease perception. Indeed, the interviews clearly show that during the RCT, the everyday life of the participants changed significantly, and the participants became more social and physical active. Nevertheless, since the Hawthorne effect might be most pronounced in the beginning of the trial, ${ }^{23}$ and since considerable lifestyle changes are difficult to maintain for long periods of time, these proposed trial participation effects may likely have eased over time. The fact that most of the participants did not feel any noticeable differences in their condition until weeks or months after the intervention may indicate that Hawthorne was not the dominating trial effect. Unfortunately, this remains speculative, as we did not explicitly study changes in diet, exercise or other lifestyle habits during the trial.

Overall, these combined unexpected trial participation effects may have affected the results of the RCT by bolstering empowerment and positivism, and, for some participants, by inducing reduction in symptoms, that all together, enhanced disease coping. The magnitude of these effects is not clear. Although we do not expect that the care effects differed between the two intervention groups due to the double-blind nature of the RCT, the feeling of improved care may have postponed starting new treatment-which was a key element of the primary outcome of the RCT being defined as treatment failure based on shared-decision making. ${ }^{9}$ Moreover, having high hopes of the effects of FMT which was considered an exciting, novel, alternative and 'natural' treatment contrasting the conventional (synthetic) treatment may further have contributed to a 'wait and see' approach hoping for 'natural' improvements of their condition-or even cure-before being compelled to request changes in the standard treatment. Although not evaluated in the current study, the concept of 'demand characteristics', ${ }^{24}$ where research participants seek to satisfy the perceived needs of the trialist-in this case by not asking for additional treatment-may further have affected the process of shared-decision-making in the RCT. ${ }^{25}$

Taken together, the potential discrepancies between the clinical setting and the research setting must be considered when discussing the clinical relevance of the RCT 
results. This could be done in a structured and robust manner using the opportunity for triangulation of findings, combining quantitative with qualitative data. ${ }^{26}$ Such triangulation will also be in line with the recent recommendations by the Outcome Measures in Rheumatology (OMERACT) Safety Working Group stressing that future rheumatology interventional trials should query patient satisfaction and measure not only how an individual feels and functions, but also the impact on everyday life addressing both benefits and harms. ${ }^{27}$

\section{Acceptability and ethics of future FMT trials}

FMT has previously been subject to embarrassment and social discomfort due to the somewhat unappealing (unsterile) nature of the intervention. ${ }^{28} 29$ This was not reported by the participants of this study. They had no problems discussing their RCT intervention with family and colleagues, although some participants did admit, when asked directly by the interviewer, that they found the procedure unappealing when the nature of FMT was presented to them. The thorough information provided by the trialists, the positive effects of FMT presented by the media, and the patients' perception of the treatment being natural and innovative may have contributed to the openness towards FMT. Still, this view on FMT does not necessarily reflect the opinion of most patients with PsA. In another study of patients affected by ulcerative colitis, many patients were interested in undergoing FMT, especially, if the safety and effectiveness of FMT were proven and other medical therapies had failed. ${ }^{30}$ In the current study, trial candidates were informed about other treatment options than FMT, including biologics. Still, many wanted to try FMT before the initiation of additional disease-modifying antirheumatic drugs. This could be due to their experience with MTX having failed to reduce symptoms and/or having imposed side effects. Furthermore, some patients consider MTX the most worrisome drug and are often initially terrified to use it. ${ }^{27}$ This view on the prevailing first-line antirheumatic drug may explain why the participants considered the transfer of gut bacteria a 'natural' treatment, as compared with a 'synthetic' drug, which again, might cast a favourable light on FMT.

The participants of the RCT had only limited concerns about safety and risk of adverse events following FMT despite being told about the known short-term side effects and the unknown long-term effects. One reason for this could be that the participants felt sufficiently informed about FMT and felt being in good hands when talking to the recruitment team prior to enrolment. Another explanation could be that the participants were, to varying degree, desperate to get better or were eager to support research into alternative treatment options, and consequently, neglected considering potential negative effects of their trial participation. Although this issue has been only scarcely investigated in patients with inflammatory arthritis, the phenomenon is well known among patients suffering from severe diseases with reduced life expectancy, such as amyotrophic lateral sclerosis ${ }^{31}$ and cancer, ${ }^{32}$ who may be more desperate to find a cure and less concerned about side effects of new treatment. Moreover, as mentioned above, patients with PsA who are treated with MTX are often 'used to' suffering side effects. Despite these 'nuisance' side effects of MTX having a considerable cumulative impact on quality of life, they are seen as 'the price you pay' for improvement. ${ }^{27}$ Consequently, since we only performed the FMT one time during the 26-week RCT, participants might also have considered the risk of a few days' side effects acceptable as compared with the weekly (or constant) side effects, which many experienced with MTX.

In contrast to the limited concerns about the risks of FMT, most of the participants stated that prior to the FMT intervention, they were anxious about the transplant delivery method being gastroscopic guidance. However, following the RCT intervention, they found the procedure tolerable and would have no concerns about receiving FMT in the future. In another study, patients with recurrent Clostridioides difficile infections who had at least one FMT shared this view. Indeed, when asked about their attitudes towards FMT following the procedure, the patients stated how easy it was to undergo and the lack of yukky factor. ${ }^{33}$ Interestingly, they also commented on the health system barriers to FMT, which they had encountered before receiving the FMT. This finding is in line with another study from 2014, which revealed that physicians dramatically overestimated both the intensity of patients' aversion and the degree to which the unappealing nature of FMT would restrain patients from considering the procedure. ${ }^{34}$ Hence, health professionals' erroneous belief that patients are unwilling to accept FMT may impose a barrier towards the use of FMT. More data on safety and efficacy of FMT may assist to overcome this barrier among health professionals. In addition, the implementation of less invasive FMT procedures such as capsule FMT, ${ }^{8}$ as suggested by several of the participants of the current study, would likely improve the general FMT acceptability as well. More research into potential health system barriers to FMT is needed.

Every participant of the current study supported the initiation of future trials testing the effects of FMT. This view was a result of several circumstances including FMT being deemed a tolerable procedure with limited side effects, the low perceived risk of FMT, the maintained strong beliefs in the effects of FMT, the overall positive experience of being part of a trial, and the need for new therapies of PsA. Still, this study proves that important ethical concerns should be addressed before initiating similar trials within this patient group. First of all, patients (and trialists) being desperate for improvement may hamper informed decision-making and render patients vulnerable to suggestion in the recruitment phase of the trial. ${ }^{35}$ Especially in RCTs testing new interventions, such as FMT, where long-term safety data are not yet available, the aspect of potential unknown risks must be thoroughly discussed between the trial candidate and the 
recruitment team. Other ethical concerns are related to disappointments in relation to having high hopes, and to the opposite situation, which we were not prepared for, where participants experience an unexpected, positive treatment response which may dramatically affect their social identity leading to difficulties adapting to the new situation.

\section{Limitations}

Semistructured interviews are particularly useful when investigating people's experiences, perspectives and ways of talking about their condition. ${ }^{36}$ In recent years, face-toface interviewing has been the most common interview form within the field of qualitative research. ${ }^{37}$ In this study, however, we decided to conduct telephone interviews. This choice was primarily made for practical reasons since participants were recruited from across Denmark. ${ }^{38}$ According to Sturges and Hanrahan who compared telephone interviews with face-to face interviews, telephone interviews may be an acceptable and valuable method for data collection. ${ }^{39}$ For example, studies have shown that in some circumstances people may feel safe and free to express their feelings and opinions more openly when not sitting face-to-face with the interviewer. ${ }^{38}$ Still, we could have missed information on non-verbal communication or behaviour guiding further questions into a particular theme.

An additional limitation in relation to collecting data was that we conducted the interviews at the end of the RCT and therefore the participants' trial experiences were retrospective. This could have introduced recall bias in favour of FMT due to the positive effects related to being part of the trial. Conducting the interviews around the four periods being central to the interview guide could have captured more nuanced feelings and thoughts. However, as the participants fulfilled weekly questionnaires about what they experienced following the trial intervention, they had previously reflected on some of the matters covered in the interview, which may have helped them recall their experiences in more details.

Moreover, interviews were restricted to only 10 patients who had already volunteered to enrol in an RCT testing FMT. Hence, patients who did not decide to participate in the trial were not interviewed. Therefore, our study findings reflect the perspective of RCT participants and cannot be transferred to the total PsA population. More research is needed to explore the thoughts, concerns, and potential barriers related to FMT among the wider group of people with inflammatory diseases. This is an important issue to cover in future studies to improve trial recruitment strategies and-if FMT proves to be effective and safe in patients with PsA-assist implementation of the treatment in the clinical setting. The study included only 10 out of 31 patients enrolled in the RCT. Nevertheless, as participants were consecutively enrolled in the study and there were no non-responders, we believe that the interviewees might very well represent the trial population. Overall, we found that there was sufficient repetition of ideas in data to suggest data saturation, meaning that the most important elements for patients had been captured.

\section{CONCLUSIONS}

The RCT influenced the participants' perception of disease and induced positive changes in their everyday life. FMT was deemed acceptable and safe, and-despite the fact that increased disease activity resulted in several intervention failures-all participants still supported more research into the field of microbiota-targeted interventions in rheumatic diseases. Importantly, due to the observed trial participation effects, discrepancies between the clinical setting and the research setting should be considered when discussing the clinical relevance of the main findings of the RCT. Triangulation of the qualitative and quantitative results could be a way to address this issue. Further research into the potential beneficial and adverse effects of FMT in addition to exploring the magnitude and mechanisms behind FMT trial participation effects in the rheumatological setting are highly needed.

\section{Author affiliations}

${ }^{1}$ Department of Rheumatology, Odense University Hospital, Odense, Denmark ${ }^{2}$ National Institute of Public Health, University of Southern Denmark, Odense, Denmark

${ }^{3}$ Department of Gastroenterology, Odense University Hospital, Odense, Denmark ${ }^{4}$ Department of Medicine, Svendborg Hospital, Odense University Hospital, Svendborg, Denmark

${ }^{5}$ Patient Research Partner, Amsterdam, Netherlands

Acknowledgements The authors thank the patient advisers and all the participants who shared their experiences with us. Moreover, we acknowledge Robin Christensen, professor in biostatistics, for his expertise in relation to the design of the FMT trial. Finally, we thank John Mason (www.manuscript.dk) for translating the quotes of the participants from Danish to English.

Contributors MSK, STS and TE conceived the study. MSK and STS drafted the interview guide. STS conducted and transcribed the interviews. MSK, TE, JK, $\mathrm{HCH}$, HLM, JKP and CSK contributed to the RCT design, the clinical implementation, and the recruitment of participants. STS, NGA and TT-T performed the initial data analysis. MSK assisted the focused coding of the material and drafted the manuscript. The manuscript was critically revised by TE, NGA, TT-T, STS and MdW. All authors reviewed and approved the final manuscript. MSK and STS contributed equally to the paper.

Funding This work was supported by Region of Southern Denmark (16/9793), Odense University Hospital (A1456), Danish Regions (Medicinpuljen; 16/2885), University of Southern Denmark (N/A), the Danish Rheumatism Association (R129A3556), the Danish Psoriasis Research Fund (N/A), and Novartis Healthcare A/S (unrestricted grant; $\mathrm{N} / \mathrm{A}$ ).

Competing interests None declared.

Patient consent for publication Not required.

Ethics approval The RCT was approved by the Regional Committees on Health Research Ethics for Southern Denmark (S-20150080) and the Danish Data Protection Agency (15/41684). The qualitative study did not require any additional approvals on the first approval as confirmed by the Ethics Committee. SS obtained the written and verbal informed consent of each participant before conducting the interview. He also made sure that the participants had read the study information sheet sent to them prior to the interview and that they were informed about their rights as participants, that the conversation would remain confidential, and that the transcript would be anonymised.

Provenance and peer review Not commissioned; externally peer reviewed. 
Data availability statement № data are available.

Supplemental material This content has been supplied by the author(s). It has not been vetted by BMJ Publishing Group Limited (BMJ) and may not have been peer-reviewed. Any opinions or recommendations discussed are solely those of the author(s) and are not endorsed by BMJ. BMJ disclaims all liability and responsibility arising from any reliance placed on the content. Where the content includes any translated material, BMJ does not warrant the accuracy and reliability of the translations (including but not limited to local regulations, clinical guidelines, terminology, drug names and drug dosages), and is not responsible for any error and/or omissions arising from translation and adaptation or otherwise.

Open access This is an open access article distributed in accordance with the Creative Commons Attribution Non Commercial (CC BY-NC 4.0) license, which permits others to distribute, remix, adapt, build upon this work non-commercially, and license their derivative works on different terms, provided the original work is properly cited, appropriate credit is given, any changes made indicated, and the use is non-commercial. See: http://creativecommons.org/licenses/by-nc/4.0/.

\section{ORCID iDs}

Maja Skov Kragsnaes http://orcid.org/0000-0001-9641-9065

Shaun Theodor Sødergren http://orcid.org/0000-0001-5854-2142

Jens Kjeldsen http://orcid.org/0000-0001-8148-6572

Maarten de Wit http://orcid.org/0000-0002-8428-6354

Nanna Gram Ahlmark http://orcid.org/0000-0002-0539-2061

Torkell Ellingsen http://orcid.org/0000-0003-0426-4962

\section{REFERENCES}

1 Ritchlin CT, Colbert RA, Gladman DD. Psoriatic arthritis. N Engl J Med 2017;376:957-70.

2 Gossec L, Smolen JS, Ramiro S, et al. European League against rheumatism (EULAR) recommendations for the management of psoriatic arthritis with pharmacological therapies: 2015 update. Ann Rheum Dis 2016;75:499-510.

3 Coates LC, Gossec L, Ramiro S, et al. New grappa and EULAR recommendations for the management of psoriatic arthritis. Rheumatology 2017;56:kew390-1253.

4 Veale DJ, Fearon U. The pathogenesis of psoriatic arthritis. Lancet 2018;391:2273-84.

5 Scher JU, Littman DR, Abramson SB. Microbiome in inflammatory arthritis and human rheumatic diseases. Arthritis Rheumatol 2016;68:35-45.

6 Salem F, Kindt N, Marchesi JR, et al. Gut microbiome in chronic rheumatic and inflammatory bowel diseases: similarities and differences. United European Gastroenterol J 2019;7:1008-32.

7 Van de Wiele T, Van Praet JT, Marzorati M, et al. How the microbiota shapes rheumatic diseases. Nat Rev Rheumatol 2016;12:398-411.

8 Ramai D, Zakhia K, Ofosu A, et al. Fecal microbiota transplantation: donor relation, fresh or frozen, delivery methods, cost-effectiveness. Ann Gastroenterol 2019;32:30-8.

9 Kragsnaes MS, Kjeldsen J, Horn HC, et al. Efficacy and safety of faecal microbiota transplantation in patients with psoriatic arthritis: protocol for a 6-month, double-blind, randomised, placebocontrolled trial. BMJ Open 2018;8:e019231.

10 Lewin S, Glenton C, Oxman AD. Use of qualitative methods alongside randomised controlled trials of complex healthcare interventions: methodological study. BMJ 2009;339:b3496.

11 Kuper A, Reeves S, Levinson W. An introduction to reading and appraising qualitative research. BMJ 2008;337:a288.

12 O'Cathain A, Thomas KJ, Drabble SJ, et al. What can qualitative research do for randomised controlled trials? A systematic mapping review. BMJ Open 2013;3:e002889.

13 Tong A, Sainsbury P, Craig J. Consolidated criteria for reporting qualitative research (COREQ): a 32-item checklist for interviews and focus groups. Int J Qual Health Care 2007;19:349-57.

14 O'Brien BC, Harris IB, Beckman TJ, et al. Standards for reporting qualitative research: a synthesis of recommendations. Acad Med 2014;89:1245-51.
15 Boyatzis RE. Transforming qualitative data: thematic analysis and code development. Thousand Oaks, California: SAGE publication, 1998.

16 Cornish F, Gillespie A, Zittoun T. Handbook of qualitative data analysis. Thousand Oaks, California: SAGE publication, 2013.

17 Bury M. Chronic illness as biographical disruption. Sociol Health IIIn 1982;4:167-82.

18 Rodrigues FB, Ferreira JJ. Strategies to minimize placebo effects in research investigations. Int Rev Neurobiol 2020;153:49-70.

19 McCambridge J, Kypri K, Elbourne D. Research participation effects: a skeleton in the methodological cupboard. J Clin Epidemiol 2014;67:845-9.

20 Finniss DG. Placebo effects: historical and modern evaluation. Int Rev Neurobiol 2018;139:1-27.

21 Colloca L, Barsky AJ. Placebo and nocebo effects. N Engl J Med 2020;382:554-61.

22 Kravvariti E, Kitas GD, Mitsikostas DD, et al. Nocebos in rheumatology: emerging concepts and their implications for clinical practice. Nat Rev Rheumatol 2018;14:727-40.

23 McCambridge J, Witton J, Elbourne DR. Systematic review of the Hawthorne effect: new concepts are needed to study research participation effects. J Clin Epidemiol 2014;67:267-77.

24 McCambridge J, de Bruin M, Witton J. The effects of demand characteristics on research participant behaviours in Non-Laboratory settings: a systematic review. PLoS One 2012;7:e39116.

25 Mathijssen EGE, Vriezekolk JE, Popa CD, et al. Shared decision making in routine clinical care of patients with rheumatoid arthritis: an assessment of audio-recorded consultations. Ann Rheum Dis 2020;79:170-5.

26 Sedgwick P, Greenwood N. Understanding the Hawthorne effect. BMJ 2015:351:h4672.

27 Andersen KM, Kelly A, Lyddiatt A, et al. Patient perspectives on DMARD safety concerns in rheumatology trials: results from inflammatory arthritis patient focus groups and OMERACT Attendees discussion. J Rheumatol 2019;46:1168-72.

28 Zipursky JS, Sidorsky TI, Freedman CA, et al. Patient attitudes toward the use of fecal microbiota transplantation in the treatment of recurrent Clostridium difficile infection. Clin Infect Dis 2012:55:1652-8.

29 Kahn SA, Gorawara-Bhat R, Rubin DT. Fecal bacteriotherapy for ulcerative colitis: patients are ready, are we? Inflamm Bowel Dis 2012;18:676-84.

30 Kahn SA, Vachon A, Rodriquez D, et al. Patient perceptions of fecal microbiota transplantation for ulcerative colitis. Inflamm Bowel Dis 2013:19:1506-13.

31 DasMahapatra P, Raja P, Gilbert J. Clinical trials from the patient perspective: survey in an online patient community. BMC Health Serv Res;27:166

32 Murphy M, McCaughan E, Carson MA, et al. Nothing to lose: a grounded theory study of patients' and healthcare professionals' perspectives of being involved in the consent process for oncology trials with non-curative intent. BMC Palliat Care 2020;19:166.

33 Pakyz AL, Moczygemba LR, VanderWielen LM, et al. Fecal microbiota transplantation for recurrent Clostridium difficile infection: the patient experience. Am J Infect Control 2016;44:554-9.

34 Zipursky JS, Sidorsky TI, Freedman CA, et al. Physician attitudes toward the use of fecal microbiota transplantation for the treatment of recurrent Clostridium difficile infection. Can J Gastroenterol Hepatol 2014;28:319-24.

35 Norris M, Poltawski L, Calitri R, et al. Hope and despair: a qualitative exploration of the experiences and impact of trial processes in a rehabilitation trial. Trials 2019;20:525.

36 Brinkmann S, Steinar K. Interviews: learning the craft of qualitative interviewing. Thousand Oaks: Sage Publications, 2015.

37 Opdenakker R. Advantages and disadvantages of four interview techniques in qualitative research. Forum Qualitative Sozialforschung/Forum: Qualitative Social Research 2006;7:4.

38 Trier-Bieniek A. Framing the telephone interview as a participantcentred tool for qualitative research: a methodological discussion. Qualitative Research 2012;12:630-44.

39 Sturges JE, Hanrahan KJ, Telephone C. Comparing telephone and face-to-face qualitative interviewing: a research note. Qualitative Research 2004:4:107-18. 